\title{
High-order essentially local extremum diminishing schemes for environmental flows
}

\author{
Yu-Heng Tseng ${ }^{*} \dagger$ \\ Department of Atmospheric Sciences, National Taiwan University, No. 1, Sec. 4 Roosevelt Rd, Taipei 106, Taiwan
}

\begin{abstract}
SUMMARY
The accuracy of numerical simulation is greatly affected by the applied convective schemes for high Reynolds number environmental flows. The convection for any scalar equation should theoretically be a purely transport process. Simple monotonic convective schemes produce smooth and stable solutions. However, the results are not reliable and possibly introduce excessive artificial diffusion (AD). We develop a new high-order monotonic formulation of essentially local extremum diminishing (ELED) schemes, using a simple algorithm to minimize the AD introduced by the convective schemes. The high-order scheme is based on the Quadratic Upstream Interpolation for Convective Kinematics formula in order to compare the results with Simple High-Accuracy Resolution Program. The resulting algorithm is applied to a two-dimensional standing interfacial wave problem and extended to three-dimensional turbulent coastal upwelling flows using large eddy simulation (LES). These examples allow sharp density interfaces in the domain, which may cause severe dispersion errors when high-order schemes are used, whereas most monotonic schemes produce significant AD. When a finer grid is used, the AD based on the Jameson, Schmidt and Turkel formula (Int. J. Comput. Fluid Dyn. 1995; 4:171-218) drops dramatically. The new high-order ELED schemes exhibit comparable accuracy to other high-order monotonic schemes and introduce less diffusion in the unsteady environmental flow simulations. The current results of LES show that the energy transfer inherent in the monotonic convection schemes may contribute non-negligibly to the turbulence. It is found that a dynamic-mixed model is able to compensate the effect of the convection schemes and provides correct flow behaviors in reality. Copyright (C) 2008 John Wiley \& Sons, Ltd.
\end{abstract}

Received 10 August 2007; Revised 20 November 2007; Accepted 20 November 2007

KEY WORDS: essentially local extremum diminishing scheme (ELED); high order; sharp interface; environmental flows; interfacial waves; upwelling; subfilter scale

\footnotetext{
${ }^{*}$ Correspondence to: Yu-Heng Tseng, Department of Atmospheric Sciences, National Taiwan University, No. 1, Sec. 4 Roosevelt Rd, Taipei 106, Taiwan.

${ }^{\dagger}$ E-mail: yhtseng@as.ntu.edu.tw

Contract/grant sponsor: National Science Council; contract/grant numbers: NSC962628M002010, NSC962745 M002003
}

Copyright (C) 2008 John Wiley \& Sons, Ltd. 


\section{INTRODUCTION}

The accuracy of numerical simulation in computational fluid dynamics may be significantly affected by the discretization scheme chosen for the convective terms. Simple central difference methods introduce propagating numerical dispersion terms (odd-order derivatives), which may generate large regions of the flow with unphysical oscillations. High-order interpolation schemes have been successful in eliminating artificial diffusion (AD), while minimizing numerical dispersion. Physical diffusion (PD) is the scattering of any scalar over time and space, e.g. the smoothing of heat between a hot surface cooling in a colder environment. Artificial (i.e. numerical) diffusion is the numerical truncation error that generates the same effects of physical diffusion artificially and should be distinguished. Artificial (numerical) dissipation is similar to the influence of numerical diffusion but occurs in momentum transfer.

The QUICK (Quadratic Upstream Interpolation for Convective Kinematics) scheme, exemplified in the steady-state multidimensional case, has demonstrated several superior properties in eliminating numerical diffusion and producing low dispersion error [1]. However, the higherorder dispersion terms may still cause overshoots and a few oscillations under highly convective conditions, even in the one-dimensional flow. In certain turbulent flows, turbulent transport variables (such as eddy viscosity) are computed as part of the solution procedure and the overshoots or undershoots may produce negative values, thus resulting in violent non-linear instability [2]. Leonard [3] has made several attempts to suppress these unphysical properties. SHARP (Simple High-Accuracy Resolution Program), based on piecewise linear characteristics, is a monotonic version of the QUICK scheme. This scheme [3] introduces exponential upwinding in regions of sharp density gradient while preserving the high-order accuracy of QUICK and eliminating extraneous overshoots (or undershoots). However, it still allows small amplitude oscillations in the solution of problems with discontinuous gradients in complex multidimensional flows [4]. It is well known that both QUICK and SHARP are designed for steady flows. A non-oscillatory version of QUICK (ULTRA-SHARP) applies a universal flux limiter to guarantee tight resolution of discontinuities without overshoot and undershoot for the unsteady flows . However, the scheme reduces to only first-order accuracy in the monotonic range of the normalized variable diagram [3], thus not maintaining the third-order properties of QUICK in certain flow regions. The restriction is more stringent in more complicated multidimensional flows. These considerations motivate the development of an alternative high-order monotonic scheme for three-dimensional flows.

Another class of monotonic schemes has been developed by Jameson [5]. These schemes are based on the local extremum diminishing (LED) property, which ensures that the solution satisfies a discrete maximum principle, thereby precluding spurious oscillations. Maxima should not increase and minima should not decrease. This principle is imposed from one time step to the next step and can be readily extended for multidimensional problems, whereas it ensures the total variation diminishing (TVD) property for one-dimensional problems [6]. It is well known that schemes that strictly satisfy the principle of LED or TVD fall back to first-order accuracy at extrema even when higher-order accuracy is achieved elsewhere [5]. This problem can be circumvented by relaxing the LED requirement to the essentially local extremum diminishing (ELED) property. These are schemes for which, in the limit as the mesh width $\delta x$ approaches zero, maxima are non-increasing and minima are non-decreasing. Jameson [5] developed a systematic procedure for the design of a broad class of ELED schemes that satisfy monotonicity constraints on both structured and unstructured grids. These schemes can be modified to improve both accuracy and multigrid convergence. 
This study explores the use of this class of schemes for environmental flows. In realistic ocean simulations, the convective fluxes are not explicitly constrained, but instead, rely on the convergence of the numerical schemes to a sufficient level of accuracy. Several results indicate that numerical models can manifest unphysically large amounts of mixing due to numerical truncation errors $[7,8]$. Smooth solutions from numerical simulations of Navier-Stokes $(\mathrm{N}-\mathrm{S})$ equation/scalar equation may generate a large amount of numerical dissipation/diffusion without careful control [8]. These effects may exacerbate while internal wave and turbulent mixing become important in the nearshore region. The $\mathrm{AD}$ introduced by numerical schemes can mask the physical effects significantly. Therefore, an accurate representation of convection is necessary to produce correct results for environmental flows [7-9].

The purpose of this study is to develop new high-order ELED schemes and provide a very simple algorithm for multidimensional convection, minimizing the AD. The schemes are based on the widely used alternative to centered differencing in ocean modeling, the QUICK formula. It provides the basis for comparison with previous studies [10-13]. The new scheme eliminates any possibility of overshoot or oscillation in QUICK by introducing the Jameson, Schmidt and Turkel (JST) and symmetric-limited positive (SLIP) schemes when needed. It switches to JST or SLIP if a local extremum occurs and thus ensures that the coefficients of the discrete approximation are non-negative, but elsewhere it still preserves the higher-order spatial accuracy. In fact, fourth-order central difference is preferred as the base for further application to minimize the inherent AD due to QUICK scheme. A general procedure for constructing fully conservative fourth-order scheme based on central difference was derived in Morinishi et al. [14].

A two-dimensional standing interfacial wave example is used to verify the new schemes, and several comparisons are made between AD and PD. This is the first time SLIP schemes have been introduced for unsteady environmental flow. In particular, the JST scheme shows dramatic reduction of $\mathrm{AD}$ over other tested schemes when the grid resolution is fine enough. The new highorder Q-JST scheme performs better than other monotonic schemes overall. The new schemes have also been used to large eddy simulation (LES) of three-dimensional upwelling flows that simulate the laboratory experiment [15] with two-layer stratification. Very sharp density gradients exist in the flow domain, and small amplitude oscillations still occur near the sharp interface when using the SHARP formula [10] even though SHARP is less oscillatory than QUICK. This flow is actually a stringent test for monotonic schemes. Correct wavelengths resulting from the growth of baroclinic instability are predicted in the current simulation. This particular flow demonstrates that the new scheme still preserves the high-order accuracy, whereas lower-order monotonic schemes are too diffusive to suppress the growth of the instability so that we cannot obtain correct wave numbers.

In LES, it has been shown by Ghosal [16] that numerical errors are usually of the same order or greater than the subgrid-scale (SGS) terms for most of the wave numbers. No theoretical frameworks for quantifying the errors in generally complex, unsteady turbulence exist at the present time [16]. In this paper, we also attempt to assess the significance of AD errors for the LES of upwelling flows by comparing their magnitudes with those of the non-linear and subgrid terms in $\mathrm{N}-\mathrm{S}$ equations. The current evaluation of $\mathrm{AD}$ based on local net fluxes is not exact; however, the comparison provides a clue for the non-negligible effect in LES. It is important to find that the dynamic-mixed model (DMM) [17] is able to provide correct energy transfer better than that of the Smagorinsky model, which may be too dissipative to initialize baroclinic instability. The dynamic subfilter model may compensate the effect of intrinsic numerical dissipation and provide an interactive mechanism between the subfilter-scale (SFS) model and the adapted monotonic 
schemes locally. The SFS model is linked to the truncation error of the discretization. The link between physical and numerical SFS modeling is still not clear. Further investigation of the turbulent interaction is needed but is beyond the scope of this paper.

The main objective of this study is to develop new high-order ELED schemes and provide a simple algorithm for multidimensional environmental flows. This paper is organized as follows. Section 2 introduces the governing equations and numerical formulation for the test cases. The new high-order ELED schemes for convection are laid out. Section 3 compares several monotonic schemes and quantifies the AD with respect to the physical one using a two-dimensional standing interfacial wave simulation. Section 4 extends the new schemes to three-dimensional laboratory upwelling flows and makes some comparisons. Finally, conclusions are drawn in Section 5.

\section{NUMERICAL METHOD}

A two-dimensional interfacial wave problem and a three-dimensional LES of coastal upwelling flows are used to illustrate the new high-order ELED treatment. In this section, we introduce the governing equations and numerical formulation briefly. Sections 2.1 and 2.2 provide the governing equations for the two-dimensional problem and LES, respectively. Section 2.3 describes the numerical discretization, including the convective formulation to construct the new ELED modification of QUICK schemes.

\subsection{Governing equations}

In order to model the flows of interest, we consider the three-dimensional, unsteady, incompressible $\mathrm{N}-\mathrm{S}$ equations with Coriolis force in Boussinesq form and the continuity equation. When writing these governing equations in conservative form, we obtain

$$
\begin{gathered}
\frac{\partial u_{j}}{\partial x_{j}}=0 \\
\frac{\partial u_{i}}{\partial t}+\frac{\partial}{\partial x_{j}}\left(u_{i} u_{j}+p \delta_{i j}-v \frac{\partial u_{i}}{\partial x_{j}}\right)=-g \rho^{\prime} \delta_{i 3}+2 \Omega\left(-u_{1} \delta_{i 2}+u_{2} \delta_{i 1}\right)
\end{gathered}
$$

where $\rho^{\prime}=\left(\rho_{*}-\rho_{\mathrm{b}}\right)$ is a perturbated relative density from Boussinesq approximation and is the deviation from the background density $\left(\rho_{\mathrm{b}}\right)$ due to the fluid motion. $\rho_{*} \ll 1$ is the relative density deviated from the reference state, defined as $\rho_{*}=\left(\rho / \rho_{0}-1\right)$, where $\rho_{0}$ is a constant reference density. $\rho_{\mathrm{b}}$ is the background density profile obtained from the background stratification and is only a function of the vertical coordinate. $g$ is the gravitational acceleration along the vertical direction. $p$ is the dynamic pressure defined in Zang [18], which includes the effect of the static density and the centrifugal force in Boussinesq approximation.

$$
p=\rho_{0} P-\rho_{0} g\left[x_{3}+\int_{0}^{x_{3}} \rho_{\mathrm{b}}\left(x_{3}^{\prime}\right) d\left(x_{3}^{\prime}\right)\right]+\frac{1}{2} \rho_{0} \Omega\left(x_{1}^{2}+x_{2}^{2}\right)
$$

where $v=\mu / \rho_{0}$ is the kinematic viscosity. The Einstein convention is used to imply summation over repeated indices, and the direction of gravity is defined in the negative $x_{3}$ direction for 
convenience. Furthermore, the density is transported via the scalar transport equation.

$$
\frac{\partial \rho}{\partial t}+\frac{\partial}{\partial x_{j}}\left(\rho u_{j}\right)=\frac{\partial}{\partial x_{j}}\left(\kappa \frac{\partial \rho}{\partial x_{j}}\right)
$$

where $\kappa$ is the molecular diffusivity.

\subsection{Filtered governing equations}

In LES, the full turbulent field is divided into a set of large-scale or 'resolved' eddies and the small-scale 'subfilter' eddies. Only the resolved eddies are computed directly, whereas the net effect of the subfiltered eddies is represented by a subfilter model. Each flow variable $v$ is decomposed into a large-scale component $\bar{v}$ and an SFS component $v^{\prime}$

$$
v=\bar{v}+v^{\prime}
$$

Following the work of Zang et al. [17], the governing equations are filtered spatially to solve the large-scale motion. The filtered equations for Equations (1), (2) and (4) become

$$
\begin{gathered}
\frac{\partial \bar{u}_{j}}{\partial x_{j}}=0 \\
\frac{\partial \bar{u}_{i}}{\partial t}+\frac{\partial}{\partial x_{j}}\left(\bar{u}_{i} \bar{u}_{j}+\bar{p} \delta_{i j}-v \frac{\partial \bar{u}_{i}}{\partial x_{j}}\right)=-g\left(\bar{\rho}^{\prime}\right) \delta_{i 3}+2 \Omega\left(-\bar{u}_{1} \delta_{i 2}+\bar{u}_{2} \delta_{i 1}\right)-\frac{\partial \tau_{i j}}{\partial x_{j}} \\
\frac{\partial \bar{\rho}}{\partial t}+\frac{\partial}{\partial x_{j}}\left(\bar{\rho}_{j}\right)=\frac{\partial}{\partial x_{j}}\left(\kappa \frac{\partial \bar{\rho}}{\partial x_{j}}\right)-\frac{\partial \chi_{j}}{\partial x_{j}}
\end{gathered}
$$

These equations govern the evolution of the large, energy-carrying scales of motions. Two extra terms that represent the effect of the unresolved, or SFS, on the resolved part of turbulence also appear in the above equations. The two terms are the SFS stress tensor $\tau_{i j}$ and the SFS flux vector $\chi_{j}$, which are defined as

$$
\begin{aligned}
\tau_{i j} & ={\overline{u_{i} u}}_{j}-\bar{u}_{i} \bar{u}_{j} \\
\chi_{j} & =\overline{u_{j} \rho}-\bar{u}_{j} \bar{\rho}
\end{aligned}
$$

Here, we use a dynamic SFS model (i.e. DMM) with local averaging for computing the influence of these small scales [17,19]. This model predicts the correct asymptotic behavior near the boundaries and allows energy backscatter and it is used successfully for many flows. Note that the LES requires a filter size that may differ from the discrete grid size; thus, the SFS stress may differ from the SGS stress [20]. In particular, the DMM computes the total SFS flux, which comprises the resolved SFS flux (the Leonard term) and the actual SGS flux [20]. In this study, a Smagorinsky SGS model [21] is also examined for comparison.

\subsection{Numerical formulation}

This paper consists of a two-dimensional interfacial wave problem and a three-dimensional LES of coastal upwelling. Both simulations share the same numerical formulation except the SFS model for the LES and a modification of pressure Poisson solver to improve temporal accuracy for the 
interfacial wave [22]. In order to deal with complex flows associated with irregular boundaries, the N-S equations (1), (2), (4) and the filtering equations (6)-(8) are transformed into curvilinear coordinates, whereas the Cartesian velocity components are retained. This is done to avoid extra source terms that arise when the velocity components are transformed [23]. Further numerical details can be found in Zang et al. [24], Tseng and Ferziger [12, 25] and Fringer et al. [13]. Here, we describe the formulation briefly and focus mostly on the new ELED modification.

2.3.1. Temporal discretization. The method of fractional steps, which splits the numerical operators and enforces continuity by solving a pressure Poisson equation, is adopted in this study. We use the improved projection method [26, 27], and advance Equation (7) without the pressure to obtain the predicted velocity field. The implicit viscous terms are advanced with the Crank-Nicolson method and the explicit viscous and source terms are advanced with the second-order Adams-Bashforth method.

2.3.2. Spatial discretization. All the spatial derivatives are approximated with second-order central differences except for the convective terms. The convective terms are discretized using the QUICK scheme [1] in which the velocity field on the cell faces is computed from the nodal values using a quadratic interpolation scheme. For the scalar transport equation, we prescribe the nearly 'twolayer' stratified flow system with a lighter fluid on top of a heavier fluid initially, resembling the density stratification in the laboratory experiment [15]. Therefore, very sharp density gradients exist in the flow domain. The convective terms in the scalar transport equation are discretized using the new schemes described in the next section in order to treat convection with steep gradients accurately. This strategy is also useful for the study of the internal wave field [28, 29].

2.3.3. Second-order ELED modification of QUICK. Jameson [5] develops systematic procedures for the design of scalar discretization schemes, which guarantee the preservation of positivity and monotonicity in the solution. To construct ELED schemes with the QUICK formula [1], we introduce the following notation for the convective discretization:

$$
\frac{\partial \rho_{j}}{\partial t}=f_{j+1 / 2}-f_{j-1 / 2}
$$

in which the time derivative is treated by the scheme described above. $f_{j+1 / 2}\left(f_{j-1 / 2}\right)$ is the numerical flux at the interface $j+\frac{1}{2}\left(j-\frac{1}{2}\right)$ between cells $j$ and $j+1(j-1)$. For a lower-order three-point scheme, $f_{j+1 / 2}\left(f_{j-1 / 2}\right)$ typically has the form

$$
\begin{aligned}
& f_{j+1 / 2}=\frac{1}{2}\left(C\left(\rho_{j}\right)+C\left(\rho_{j+1}\right)\right)-d_{j+1 / 2} \\
& f_{j-1 / 2}=\frac{1}{2}\left(C\left(\rho_{j-1}\right)+C\left(\rho_{j}\right)\right)-d_{j-1 / 2}
\end{aligned}
$$

where $C\left(\rho_{j}\right)$ represents the convective term discretized at cell $j . d_{j+1 / 2}$ and $d_{j-1 / 2}$ are the dissipative terms introduced in order to eliminate the oscillation produced from the numerical simulation. For a higher-order scheme, $f_{j+1 / 2}$ will depend on a larger stencil. Higher-order nonoscillatory schemes can be derived by introducing anti-diffusive terms in a controlled manner.

One approach is the JST scheme [30] for which

$$
d_{j+1 / 2}=\eta_{j+1 / 2}^{(2)} \Delta \rho_{j+1 / 2}-\eta_{j+1 / 2}^{(4)}\left(\Delta \rho_{j+3 / 2}-2 \Delta \rho_{j+1 / 2}+\Delta \rho_{j-1 / 2}\right)
$$


We use variable coefficients $\eta_{j+1 / 2}^{(2)}, \eta_{j+1 / 2}^{(4)}$ to produce a low level of diffusion in regions where the solution is smooth but prevent oscillations near discontinuities. The JST scheme is inexpensive and robust but tends to be excessively dissipative in boundary layers. It has been proved that the JST scheme with appropriately chosen coefficients is both second-order accurate at smooth extrema and is ELED [5].

Set

$$
\begin{gathered}
\eta_{j+1 / 2}^{(2)}=\frac{1}{2}\left|u_{j+1 / 2}\right| q_{j+1 / 2} \\
\eta_{j+1 / 2}^{(4)}=\frac{1}{4}\left|u_{j+1 / 2}\right|\left(1-q_{j+1 / 2}\right)
\end{gathered}
$$

where $q_{j+1 / 2}=R\left(\Delta \rho_{j+3 / 2}, \Delta \rho_{j-1 / 2}\right)$. Define $R(m, n)$ as

$$
R(m, n)=\left|\frac{m-n}{\max \left(|m|+|n|, \varepsilon \Delta x^{r}\right)}\right|^{k}
$$

where $\varepsilon>0$ and $r=2, k=3$. The scheme is LED when $\varepsilon=0$ and ELED when $\varepsilon>0$. The relaxation of Equation (17) when $\varepsilon>0$ yields second-order accuracy for smooth extrema [5].

An alternative approach is to convert a first-order accurate monotone scheme to a higher-order scheme by adding limited amounts of anti-diffusive flux to prevent spurious oscillations. The limited anti-diffusive terms result in a general family of SLIP schemes to guarantee the positivity. In fact, the JST scheme can be classified as a member of SLIP schemes as well. In this scheme, the third-order diffusion defined by Equation (14) is modified by the inserting limiters, which produce an equivalent three-point scheme with positive coefficients:

$$
d_{j+1 / 2}=\eta_{j+1 / 2}^{(2)} \Delta \rho_{j+1 / 2}-L\left(\eta_{j+3 / 2}^{(2)} \Delta \rho_{j+3 / 2}, \eta_{j-1 / 2}^{(2)} \Delta \rho_{j-1 / 2}\right)
$$

where $L(m, n)$ represents a variety of flux limiters and satisfies the LED scheme properties. Note that the JST scheme can also be cast as a member of the SLIP family of schemes after regrouping terms. Three other typical limiters [6] are tested in this study. Define $S(m, n)$ as

$$
S(m, n)= \begin{cases}1 & \text { if } m>0 \text { and } n>0 \\ -1 & \text { if } m<0 \text { and } n<0 \\ 0 & \text { if } m n<0\end{cases}
$$

(1) Van-leer:

$$
L(m, n)=S(m, n) \frac{2|m||n|}{|m|+|n|}
$$

(2) Minmod:

$$
L(m, n)=S(m, n) \min (|m|,|n|)
$$

(3) Superbee:

$$
L(m, n)=S(m, n) \max [\min (2|m|,|n|), \min (|m|, 2|n|)]
$$


2.3.4. Higher-order ELED-QUICK schemes. Schemes of any desired order of accuracy in which the solution does not contain extrema can be constructed by combining any high-order and lowerorder convective schemes where the lower-order scheme has positive coefficients and is LED. Here, we construct a family of high-order monotonic schemes based on the QUICK formula since QUICK is well known as a high-order scheme that allows oscillations. Define the numerical fluxes of the higher- and lower-order schemes, respectively:

$$
\begin{gathered}
f_{j+1 / 2}^{H}=f_{\mathrm{QUICK}} \\
f_{j+1 / 2}^{L}=f_{\mathrm{ELED}}
\end{gathered}
$$

where the low-order scheme has positive coefficients and is LED (JST or other flux limiters schemes). Let us define an anti-diffusive flux

$$
a_{j+1 / 2}=f_{j+1 / 2}^{H}-f_{j+1 / 2}^{L}
$$

In order to define a limited corrective flux $f_{j+1 / 2}^{C}$, let $b_{j+1 / 2}$ be a bound determined from the local slopes at node $j$. If node $j$ is not a local extremum, set $b_{j+1 / 2}=\min \left(\left|\rho_{j}-\rho_{k}\right|\right)$, where $k$ is the stencil neighboring $j$ in each direction. Otherwise, $b_{j+1 / 2}=0$. Set

$$
f_{j+1 / 2}^{C}=\operatorname{sign}\left(a_{j+1 / 2}\right) \min \left(\left|a_{j+1 / 2}\right|, \beta_{j+1 / 2} b_{j+1 / 2}\right)
$$

where $\beta_{j+1 / 2}>0$. Then the convection flux can be defined as

$$
f_{j+1 / 2}=f_{j+1 / 2}^{L}+f_{j+1 / 2}^{C}
$$

Thus, it reduces to the high-order scheme when the limiters are not active. The limiter depends only on the magnitude of the local slopes. It has been shown that the scheme is ELED if the low-order scheme is ELED [5]. We constructed a family of higher-order ELED schemes, all of which use QUICK as the high-order scheme, as indicated in Table I. These schemes are tested in the following examples. Note that the current approach is similar to the high-order construction proposed in Jameson [5] and can be seen as a modification and extension to multidimension. However, the new scheme is devised in order to ensure that the local extrema do not increase/decrease too much for multidimension, i.e. minimize the amount of flux limiter correction. The correction is along all directions in the stencils of $j$ rather than along only one dimension. In addition, the particular choice of $b_{j+1 / 2}$ is different from that in Jameson [5].

Table I. Table of the higher-order ELED schemes based on QUICK.

\begin{tabular}{lcc}
\hline Method & Higher-order flux & Lower-order flux \\
\hline Q-JST & QUICK & JST \\
Q-Minmod & QUICK & Minmod \\
Q-Van-leer & QUICK & Van-leer \\
Q-Superbee & QUICK & Superbee \\
\hline
\end{tabular}




\section{NUMERICAL SIMULATION OF STANDING INTERFACIAL WAVES}

It is well known that breaking internal waves lead to a significant amount of turbulence, and the consequent mixing occurs in stratified fluids via instabilities. The interfacial wave motion is an important process in turbulent mixing in coastal waters. The convective schemes in numerical models can manifest unphysically large amounts of mixing due to numerical truncation errors. All of the monotonic convection schemes introduce $\mathrm{AD}$, which may cause a large amount of unphysical mixing. Thus, our first example is a two-dimensional standing interfacial wave configuration, which is a typical mechanism leading to internal wave breaking and turbulence [28,31]. In this study, we quantify the numerical diffusion introduced by various convective schemes on a variety of grid sizes.

\subsection{Flow description}

The simulation domain is a two-dimensional rectangle resembling the experiments of Thorpe [32]. Standing waves are generated by initializing the domain with an initial finite amplitude standing wave profile, which is derived by Thorpe as second-order approximation for deep Boussinesq waves:

$$
\eta=a\left[1-\frac{a^{2} k^{2}}{64} \cos (k x)-\frac{a^{2} k^{2}}{8} \cos (2 k x)\right]
$$

where $k=2 \pi / L$ is the lowest horizontal wave number allowed in this domain ( $L$ : horizontal domain size) and $a$ is the wave amplitude [31,32]. The density profile is initialized with a hyperbolic tangent distribution. A detailed description of the simulation model can be found in Fringer and Street [28] and Fringer [31].

Thorpe [32] showed experimentally and theoretically that finite amplitude effects induce a shear instability for standing interfacial waves. Standing waves behave quite differently from progressive waves because maximum shear occurs near the zero deflection points (nodes) rather than the crests and troughs. We simulate one wave, and the maximum shear causes Kelvin-Helmholtz billows to form at the node located in the domain center. Without viscosity or finite interface effects, the influence of finite amplitude on dispersion (frequency $w$ ) with $\Delta \rho / \rho_{0} \ll 1$ is [32]

$$
w^{2}=\frac{g k \Delta \rho}{2 \rho_{0}}\left(1-\frac{a^{2} k^{2}}{8}\right)
$$

Finite amplitude effects tend to decrease the frequency. This results from the increase in the inertial force present in a standing wave as the amplitude increases. The current numerical code is modified from a code that has been shown by Fringer [31] to be capable of capturing the instabilities as they develop at the nodes in standing wave experiments of Thorpe [32]. The numerical formulation is described in the previous section. The accuracy and convergence tests have been illustrated in Fringer and Street [28], which show that the numerical scheme is generally second-order accurate in both space and time.

The product $k a$ is a measure of the steepness of the wave. Thorpe's results qualitatively show that at wave steepness of roughly $k a=0.2$, blurred regions become apparent at the nodes, and at $k a=0.4$ the wave begins to break down. Fringer [31] illustrated the evolution of the instabilities 

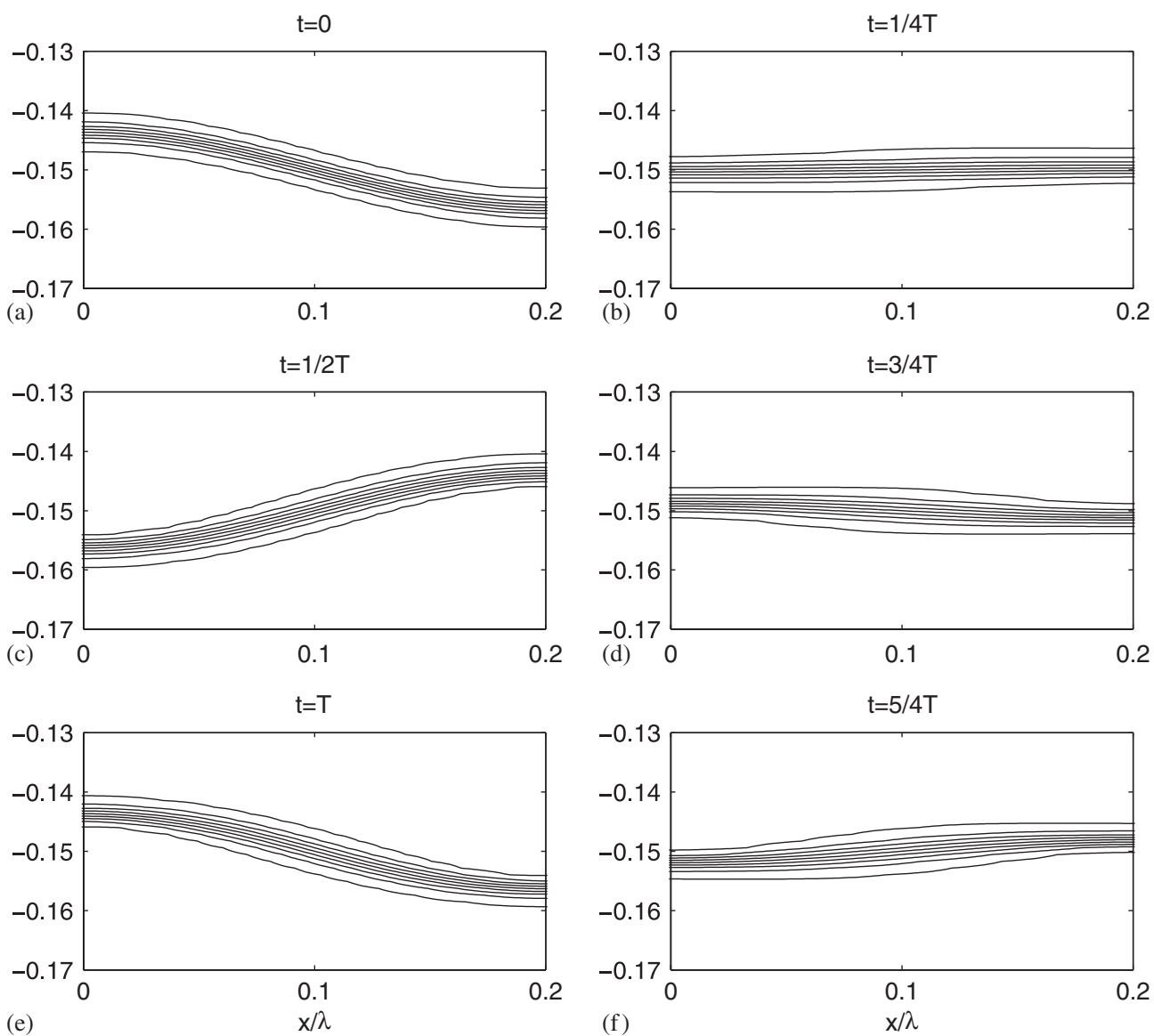

Figure 1. The evolution of the interface for a standing wave of steepness $k a=0.1$, where $T=2 \pi / w$ is the standing wave period from Equation (29). The blurring mechanism described by Thorpe [32] is evident at $t=5 / 4 T$ for this steepness, but no apparent instabilities developed.

for several $k a$ and qualitatively compared the results with those of Thorpe [32]. He also extended the numerical model to study the interfacial wave breaking [28] using three-dimensional direct numerical simulation. In our study, we focus on the comparison of the AD introduced by different convective schemes and demonstrate the benefits of the new high-order ELED methods. We set the steepness $k a=0.1$ to ensure that interfacial waves do not break, as we are more interested in the numerical scheme than the physical breaking process.

The evolution of the density contours over 5/4T for a standing wave is shown in Figure 1, where $T=2 \pi / w$ is the standing wave period from Equation (29). No apparent instability is evident, other than some slight thickening of the interface. The thickening acts to lower the gradient Richardson number $\left(R i=N^{2} /(\mathrm{d} U / \mathrm{d} z)^{2}\right.$, where $N=\sqrt{-(g / \bar{\rho}) \partial \bar{\rho} / \partial z}$ is the Brunt-Väisälä frequency) of the flow but not enough to generate any instability. The major thickening occurs near the node due to the blurring mechanism described by Thorpe [32]. 


\subsection{Simulation results}

The initial density profile (Figure 2(a)) triggers the flow oscillation, which subsequently decays. The interface becomes thicker with increasing time due to molecular diffusion. Ideally, the convective scheme should not affect the probability density function (PDF) of density distribution and introduce no false diffusion. This motivates the use of central difference schemes. However, they cause significant dispersion error near sharp interfaces, thus possibly resulting in numerical instability. The QUICK scheme provides high-order accuracy but introduces spurious oscillations and false diffusion. SHARP is a modification of QUICK, which is monotonic in the normalized variable diagram. It is well known that SHARP introduces more numerical diffusion than QUICK, as it reduces to first-order upwinding in certain flow regions. JST is a high-order monotonic scheme based on central differences. The evolutions of the introduced AD by these three methods for a vertically stretched grid of $128 \times 128$ are demonstrated in Figure 3. A schematic of the domain grid is shown in Figure 2(b) (every second grid is plotted). A fine grid is located near the center. The AD resulting from these schemes peaks when the amplitude is at a minimum. The JST scheme shows a significant reduction in unphysical diffusion on this grid configuration and, in fact, it has been proved that the $\mathrm{AD}$ due to JST is roughly proportional to $\Delta x^{3}$, whereas no explicit relationship is found between other convective schemes and the grid size. It is not an easy task to quantify AD for unsteady flows in practice. In this study, the $\mathrm{AD}$ introduced by the non-linearity at each cell is based on the numerical discretization and referenced by the central difference from which no
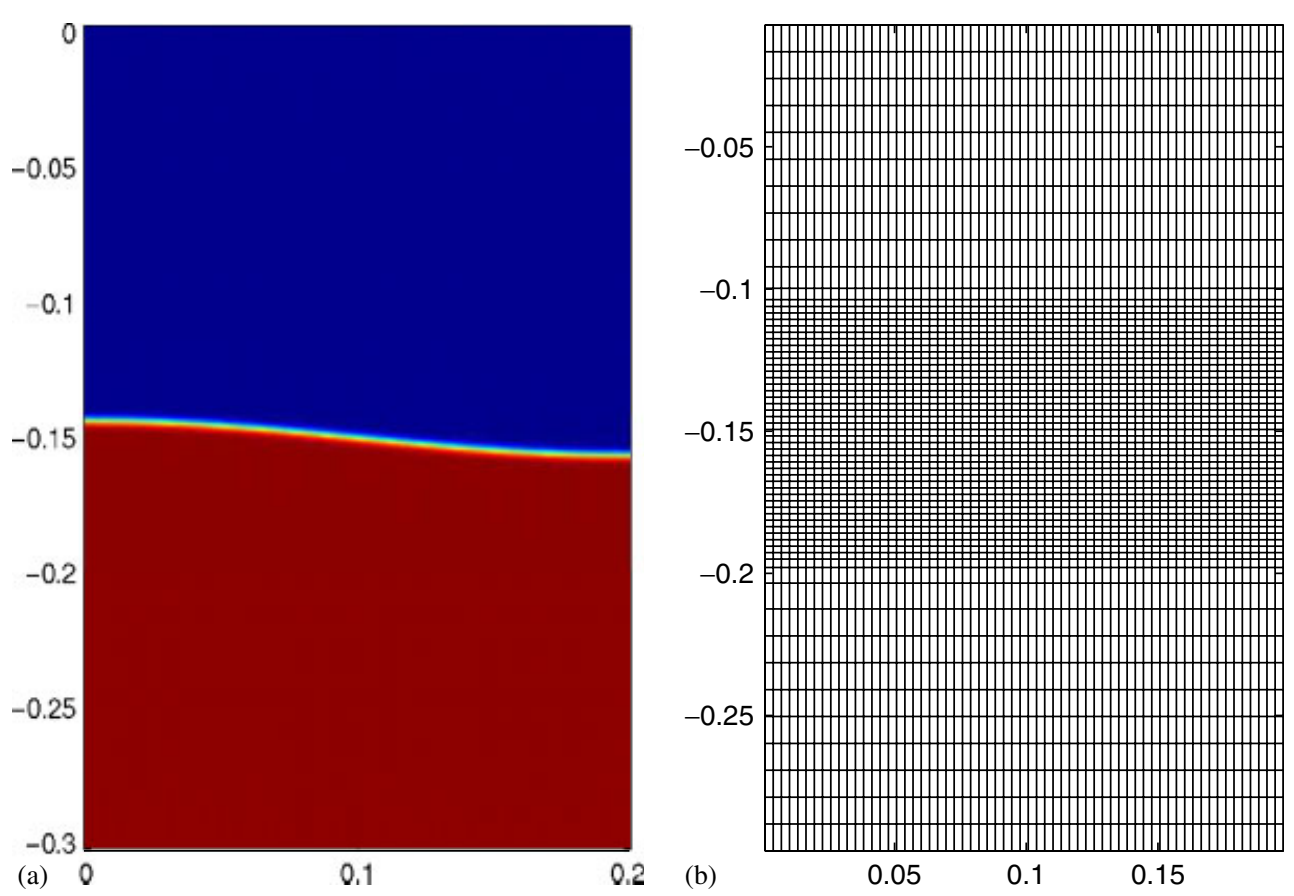

Figure 2. (a) The initial density profile of the two-dimensional standing wave experiment and (b) a vertically stretched grid for numerical simulation of standing interfacial waves (grid resolution: $128 \times 128$. Every second grid is shown). 


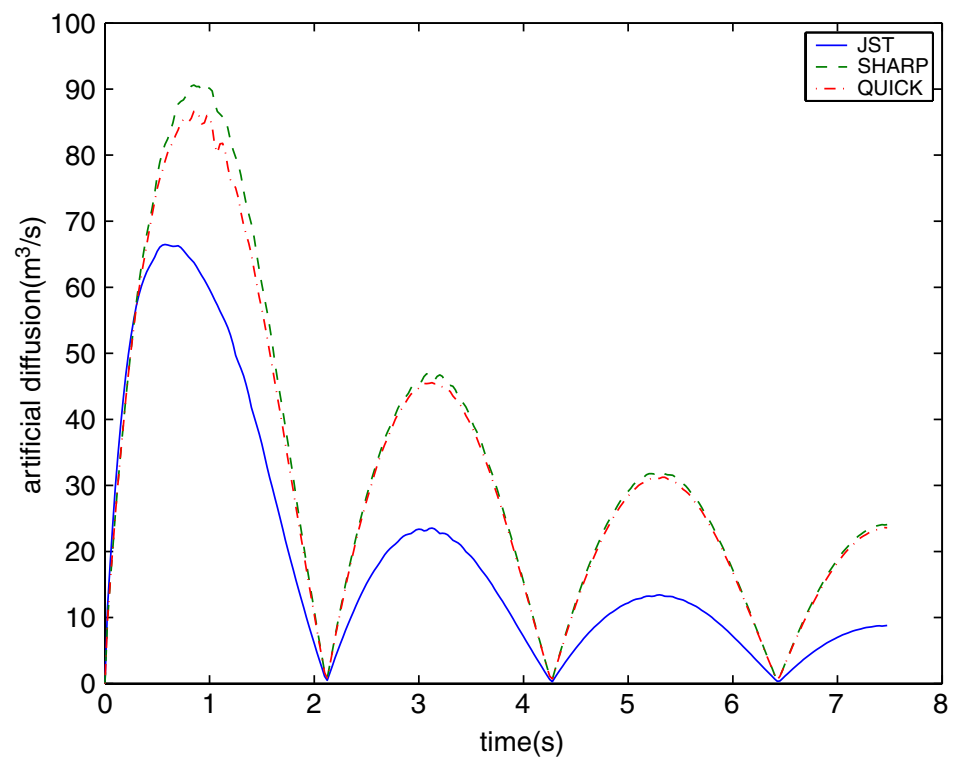

Figure 3. Time evolution of the artificial diffusion introduced by JST, SHARP and QUICK.

diffusion error occurs theoretically. It is defined as the difference between the local net volume flux calculated by the specified monotonic scheme and by central difference. In fact, it is the AD term $d_{j+1 / 2}\left(d_{j-1 / 2}\right)$ in Equations (12) and (13) for every cell as $d_{j+1 / 2}=0\left(d_{j-1 / 2}=0\right)$ implies central difference discretization. The total AD is to sum up the AD within all cells in the whole simulation domain:

$$
\mathrm{AD}=\sum_{\Omega}\left|f_{\text {scheme }}-f_{\text {central }}\right| \Delta V
$$

where $f_{\text {scheme }}$ and $f_{\text {central }}$ are the volume flux of the cell, whereas we discretize the convection term in Equation (4). The current approach does not quantify AD exactly; however, it appears to be appropriate to measure the effects of $\mathrm{AD}$ within the non-linearity terms for general unsteady, non-periodic flows. At the finer grid, less error should be generated and this quantity should be smaller.

The grid resolution studies focus on the ratio between the AD and PD instantaneously and are averaged by the phase period $T$. The PD is defined as the summation of the net volume flux of the last term in Equation (4). The average ratio of the AD to the PD is tabulated in Table II. All of the ratios decrease as the number of grid points increases. According to the comparison, using a stretched grid near the interface or at least uniform grid size of $256 \times 256$ is necessary to fully minimize the $\mathrm{AD}$ in current simulation. This requirement is case dependent and may vary with physical conditions. When a vertically stretched grid is used (grid: $128 \times 128^{\mathrm{a}}$ in Table II) in the vicinity of the interface, the ratio of AD to PD is reduced significantly. The result indicates that the false diffusion due to the convective schemes is mainly present near the sharp interface.

When the grid interval is large, the ELED schemes produce larger amount of numerical diffusion than QUICK and SHARP. JST reduces the numerical diffusion roughly by a factor of 4-6 when halving the grid interval in each direction. It is found that the JST scheme performs best 
Table II. Comparison of the averaged artificial diffusion introduced by different monotonic schemes (SHARP and standard ELED schemes with different limiters).

\begin{tabular}{lccccccc}
\hline & & \multicolumn{5}{c}{ Artificial diffusion/physical diffusion } \\
\cline { 3 - 7 } Grid size & Physical diffusion & QUICK & SHARP & JST & Minmod & Van-leer & Superbee \\
\hline $32 \times 32$ & $1.5121 \mathrm{e}-04$ & 4.675 & 6.444 & 8.011 & 9.853 & 9.019 & 8.428 \\
$64 \times 64$ & $1.0546 \mathrm{e}-04$ & 1.636 & 1.976 & 2.069 & 3.464 & 2.752 & 2.418 \\
$128 \times 128$ & $4.9386 \mathrm{e}-05$ & 0.445 & 0.457 & 0.301 & 1.053 & 0.440 & 0.752 \\
$256 \times 256$ & $3.3552 \mathrm{e}-05$ & 0.156 & 0.158 & 0.076 & 0.390 & 0.103 & 0.456 \\
$128 \times 128^{\mathrm{a}}$ & $3.2651 \mathrm{e}-05$ & 0.150 & 0.152 & 0.073 & 0.374 & 0.097 & 0.450 \\
\hline
\end{tabular}

${ }^{a}$ Vertically stretched grid.

Table III. Averaged artificial diffusion introduced by higher-order ELED schemes with different limiters.

\begin{tabular}{lcccc}
\hline & \multicolumn{4}{c}{ Artificial diffusion/physical diffusion } \\
\cline { 2 - 5 } Grid size & Q-JST & Q-Minmod & Q-Van-leer & Q-Superbee \\
\hline $32 \times 32$ & 6.177 & 7.083 & 7.118 & 6.688 \\
$64 \times 64$ & 1.937 & 2.387 & 2.123 & 2.060 \\
\hline
\end{tabular}

(significantly less diffusion) among the SLIP schemes for all grid sizes. Among the SLIP schemes with flux limiters, Superbee introduces less diffusion than the two other limiters (Minmod and Van-leer). Van-leer is comparable to the JST scheme when the grid interval is small.

When the scheme does not fully resolve the interface (small number of grid points), the numerical diffusion generated by the standard ELED schemes is relatively high and this can be remedied by the higher-order ELED schemes developed in the previous section, which still preserve the monotonic property without sacrificing the accuracy. A comparison of the average AD introduced by several higher-order ELED schemes is provided in Table III. Note that the high-order ELED schemes described here use QUICK as the based high-order method in order to compare with the performance of SHARP. The high-order schemes produce similar diffusion to SHARP, whereas the JST scheme produces slightly less diffusion than SHARP. When the grid is fine enough, JST performs better than SHARP and even better than QUICK.

\section{LABORATORY UPWELLING FLOW SIMULATIONS}

A particular objective of our present work is to evaluate the new convective schemes for complex three-dimensional environmental flows and to minimize the truncation error. We now present a comparison of LES for a three-dimensional turbulent stratified and rotating flow, which simulates the coastal upwelling phenomenon in a laboratory experiment [15].

\subsection{Flow description}

The flow geometry is a quadrant of the tank with a sloping bottom in the laboratory experiment. A schematic of the domain grid is shown in Figure 4 (every fourth grid is plotted). A $80 \times 80 \times 80$ 
grid configuration is employed, non-uniform in the radial and vertical direction but uniform in the azimuthal direction. Geometric stretching is employed in the non-uniform grid distribution. A no-slip condition for the velocity is applied to the top, side and bottom walls. Periodic boundary conditions are used in the azimuthal direction. The initial vertical density field is a hyperbolic tangent profile approximating a two-layer stratified system. There is no azimuthal and horizontal variation. A no-flux condition is used for the salinity at the solid walls, and periodic boundary conditions are applied at the two azimuthal boundaries. Initially, the stratified fluid is in solid body rotation with the container. At time $t=0$, upwelling flow is generated by relative rotation of the top lid, which simulates a wind stress on the fluid surface, see Tseng and Ferziger [12] for more model setups.

The governing equations are the grid-filtered continuity (6), N-S (7) and the scalar transport (8) equations, which are solved in the reference frame rotating with the container. The parameters of the upwelling flow simulation are matched to those in the experiments of Narimousa and Maxworthy [15] and are given in Table IV. A detailed comparison of the numerical results with laboratory experiments and a grid resolution study have been performed in Zang and Street [10] to verify the numerical algorithm for the current flow. Table $\mathrm{V}$ shows some quantitative comparisons reported in Tadepalli [11], where he characterized the instability mechanism using linear stability theory. The wave number (mode) is essentially the number of saturated frontal waves generated by the instability. Phase speed is defined as the ratio of maximum wave drift velocity $\left(u_{w}\right)$ to the disk velocity $\left(U_{\mathrm{p}}=r \Delta \Omega\right)$. The numerical simulation compares well with the

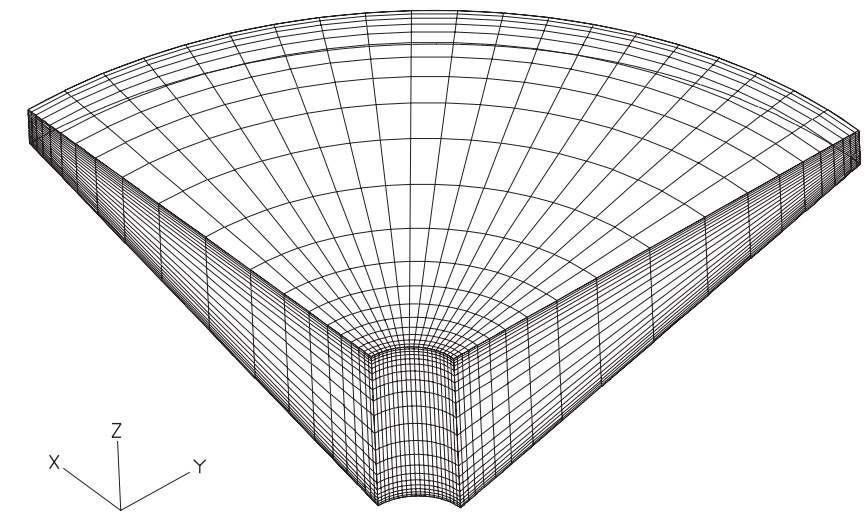

Figure 4. Grid for numerical simulation of upwelling flow (every fourth grid is shown).

Table IV. Parameters of the simulations.

\begin{tabular}{lc}
\hline Tank rotation, $\Omega\left(\mathrm{s}^{-1}\right)$ & 2.27 \\
Lid rotation, $\Delta \Omega\left(\mathrm{s}^{-1}\right)$ & 0.185 \\
Total depth, $H(\mathrm{~m})$ & 0.20 \\
Salinity diff., $\Delta \rho\left(\mathrm{kg} / \mathrm{m}^{3}\right)$ & 18 \\
Tank radius, $R_{0}(\mathrm{~m})$ & 0.45 \\
Slope & 0.27 \\
Reynolds number, $R e$ & 2995 \\
Schmidt number, $S c$ & 723 \\
\hline
\end{tabular}


Table V. Characteristics of the instabilities.

\begin{tabular}{lccc}
\hline & Linear theory & LES & Experiment \\
\hline Mode $\left(k_{\theta}\right)$ & 25 & 24 & 26 \\
Growth rate $(w)$ & 0.021 & 0.02 & N $/ \mathrm{A}$ \\
Phase speed $\left(u_{w} / U_{\mathrm{p}}\right)$ & 0.39 & 0.36 & 0.37 \\
\hline
\end{tabular}

linear stability theory prediction and the experiments of Narimousa and Maxworthy [15]. Tseng and Ferziger [12] further investigated the turbulent mixing and vorticity structures similar to those observed in the west coast of the U.S. In order to verify the new high-order ELED schemes in this study, we leave the $\mathrm{N}-\mathrm{S}$ solver unchanged except the convective discretization of the scalar transport equation. The current numerical experiment is modified from the code used in Tseng and Ferziger [12].

\subsection{Simulation results}

Even though SHARP has already removed a large portion of the oscillations resulting from QUICK formulation in solving the scalar transport equation [10], very sharp density (salinity) gradients exist (hyperbolic tangent profile vertically) initially in the flow domain, and small amplitude oscillations still occur near the sharp interface. This flow is actually a stringent test for monotonic schemes. It is unstable for a range of wavelengths, and there is a wavelength at which the growth rate is maximum. If the initial disturbance contains all wave numbers, the most unstable mode will emerge and dominate the disturbance field [11]. Accurate wavelengths resulting from the growth of baroclinic instability are predicted by SHARP and also by our new method. This example shows that the new scheme still preserves high-order accuracy, whereas low-order monotonic schemes are too diffusive to suppress the growth of instability (i.e. correct wave numbers and phase speed cannot be obtained).

Figure 5 shows such a comparison of the salinity fields at $t=1.31 t_{s}$ using the SHARP formula and the high-order ELED schemes (Q-JST). $t_{s}$ is the spin-up time [33], used as the reference time scale, defined as $t_{s}=\left(h_{10} / \Delta \Omega\right)((\Omega+\Delta \Omega) / v)^{1 / 2}$, where $\Delta \Omega$ is the differential lid angular velocity and $\Omega$ is the tank rotation angular velocity. $h_{10}$ is the initial upper layer depth. Figure 5(a) and (b) are the horizontal section at $z=0.9 \mathrm{~h}$ and show that the wave number in the azimuthal direction is roughly 24 (periodicity in $\pi / 2$ ) in both cases, which is consistent with that predicted by the linear stability theory and seen in the laboratory experiments $[11,15]$, see Table V. Both simulations produce similar flow patterns using the same initialization. In fact, this flow is very sensitive to the initial condition. As the non-linearity dominates the flow, identical initialization is necessary. The flow cannot be well resolved when the pure JST scheme is used (Figure 6) on the current grid, as pure JST may add too much AD in regions where it is not required. Note that the wave heights in the lower right and upper left corners are significantly smaller than those predicted by SHARP and the high-order Q-JST schemes (Figure 5). Similar unresolved structures are observed when other second-order TVD or pure SLIP schemes are used.

Although either SHARP or high-order Q-JST resolves the flow well and the pattern is similar to the experimental results, it is clear that SHARP is not oscillation-free in the simulation, as expected. This results from the fact that SHARP is originally designed for one-dimensional steady flows rather than multidimensional convective flows. Operator splitting could be employed to 

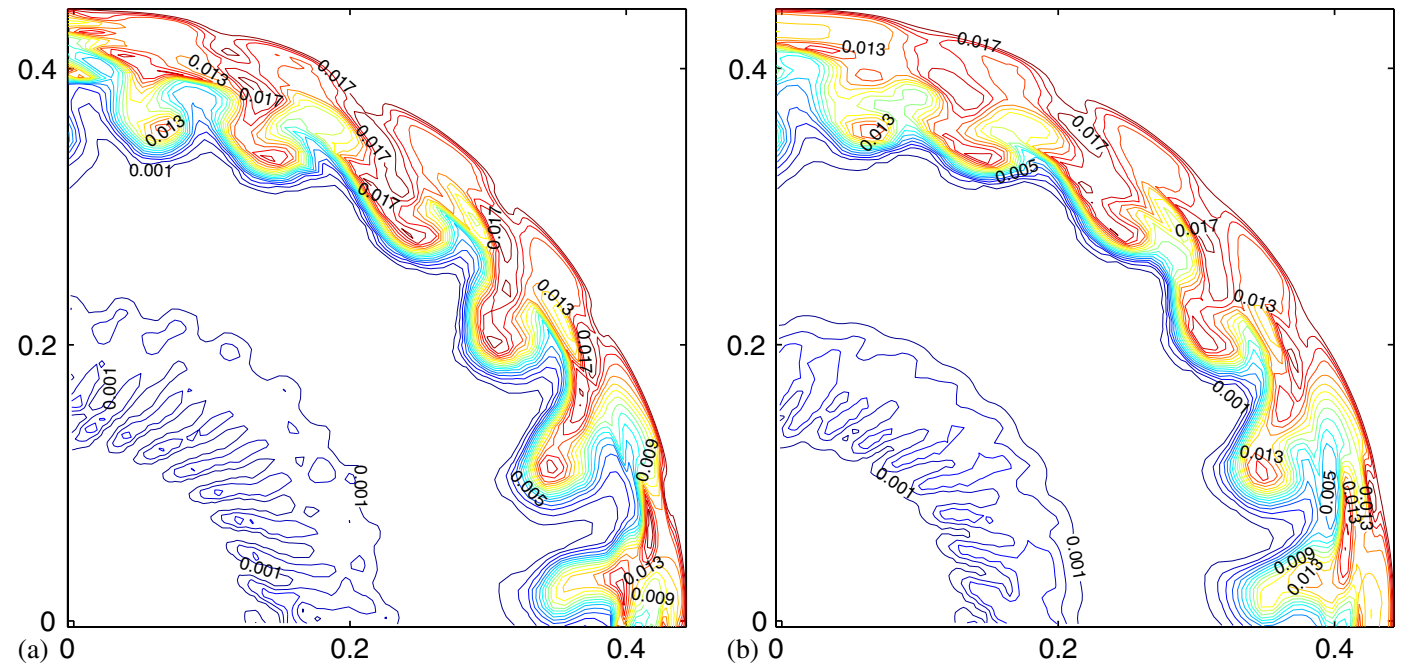

Figure 5. The comparison of salinity contours for: (a) SHARP and (b) high-order ELED scheme at $t=1.31 t_{s}$ and $z=0.9 h$.

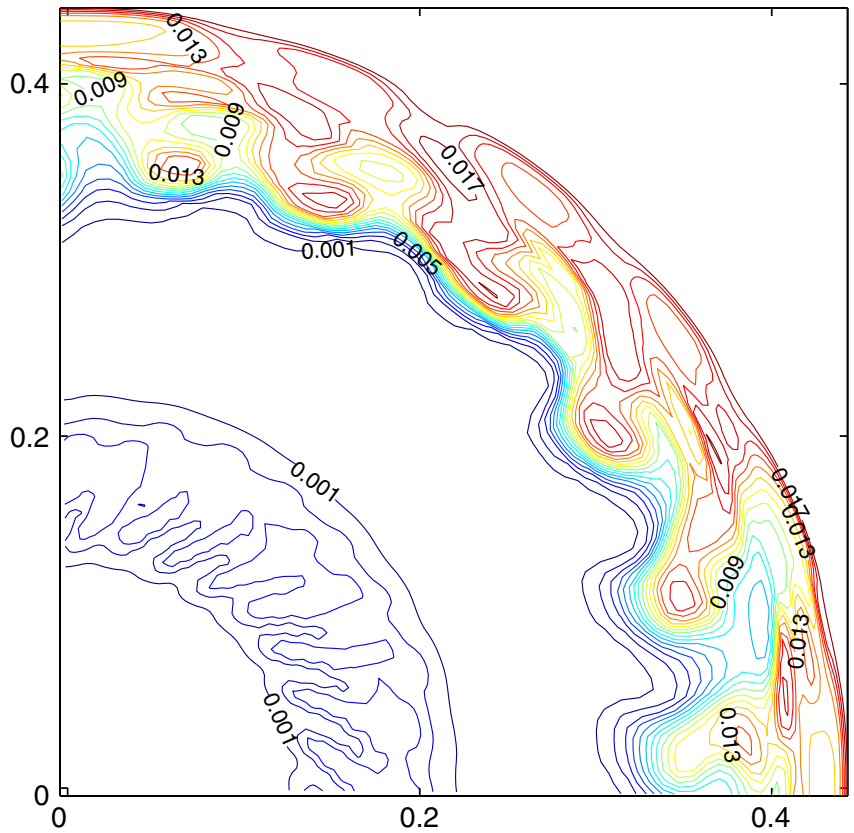

Figure 6. Salinity contours using pure JST scheme at $t=1.31 t_{s}$ and $z=0.9 h$. 

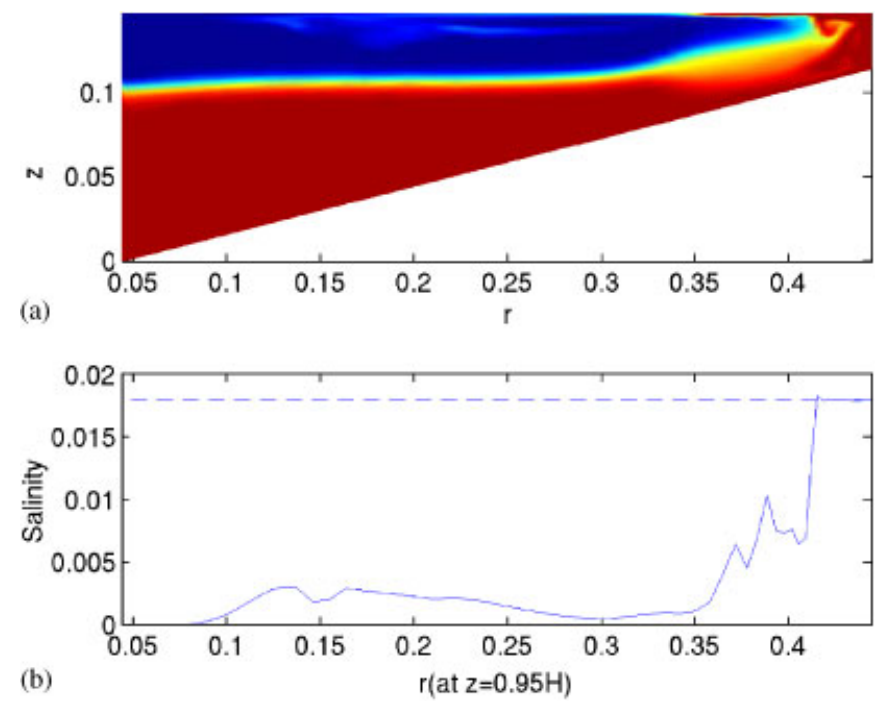

Figure 7. (a) Salinity fields in a vertical section at $\theta=80^{\circ}$ using SHARP and (b) the corresponding salinity profile at $z=0.97 \mathrm{~h}$.

Table VI. Comparison of the averaged artificial diffusion introduced by a variety of monotonic schemes (SHARP and standard ELED schemes with different limiters).

\begin{tabular}{lccccc}
\hline & \multicolumn{5}{c}{ Artificial diffusion } \\
\cline { 2 - 5 } Grid size & SHARP & Q-JST & Q-Minmod & Q-Van-leer & Q-Superbee \\
\hline $64 \times 64 \times 64$ & 0.429 & 0.332 & 0.333 & 0.330 & 0.337 \\
$80 \times 80 \times 80$ & 0.195 & 0.148 & 0.149 & 0.147 & 0.150 \\
\hline
\end{tabular}

All quantities are normalized by $v \Delta \rho / R_{0}^{2}$.

preserve monotonicity of SHARP in unsteady complex flows. Figure 7(a) is the salinity field in the vertical section at $\theta=80^{\circ}$, and Figure 7(b) is the corresponding salinity profile at $z=0.97 \mathrm{~h}$. We can see its inherent overshoot. However, the high-order ELED scheme guarantees monotonicity and completely eliminates the overshoot, while adding a similar (or even slightly smaller) amount of diffusion overall (Table VI). The AD is defined in the same way as that in the previous section. The AD resulting from the new high-order ELED schemes is qualitatively similar, indicating that the numerical property is dominated by the based high-order scheme (QUICK). The AD is insensitive to the types of limiters. The salinity fields and profile for the high-order Q-JST scheme at the same time and location of Figure 7 are shown in Figure 8. The results clearly show nonoscillatory behaviors. The non-negative, bounded salinity is important for quantifying turbulent mixing in the coastal ocean. Unphysical overshoots may potentially change the PDF distribution of salinity/temperature, thus overpredicting the estimation of mixing produced by the upwelled waters [34]. 

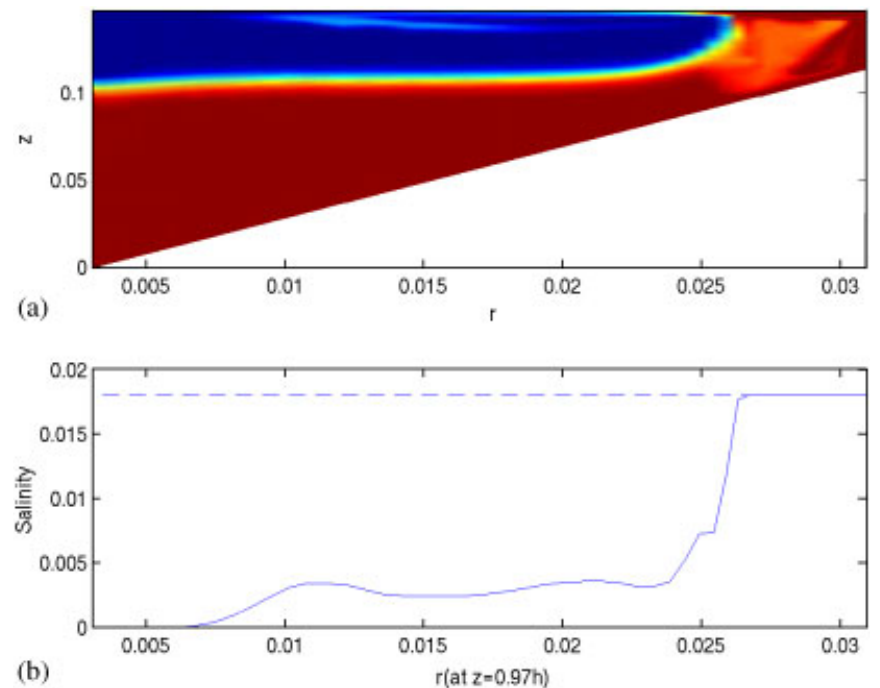

Figure 8. (a) Salinity fields in a vertical section at $\theta=80^{\circ}$ using high-order ELED scheme based on JST and (b) the corresponding salinity profile at $z=0.97 \mathrm{~h}$.

Table VII. Comparison of SFS diffusion introduced by a variety of monotonic schemes (SHARP and standard ELED schemes with different limiters).

\begin{tabular}{lcccccc}
\hline & \multicolumn{5}{c}{ SGS diffusion } \\
\cline { 2 - 7 } Grid size & SHARP & Q-JST & Q-Minmod & Q-Van-leer & Q-Superbee & Central \\
\hline $64 \times 64 \times 64$ & 0.042 & 0.030 & 0.030 & 0.030 & 0.031 & - \\
$80 \times 80 \times 80$ & 0.020 & 0.013 & 0.012 & 0.012 & 0.013 & 0.062 \\
\hline
\end{tabular}

All quantities are normalized by $v \Delta \rho / R_{0}^{2}$.

Table VIII. Comparison of the averaged artificial diffusion introduced by a variety of monotonic schemes (SHARP and standard ELED schemes with different limiters) without SFS modeling.

\begin{tabular}{lccccc}
\hline & \multicolumn{5}{c}{ Artificial diffusion } \\
\cline { 2 - 6 } Grid size & SHARP & Q-JST & Q-Minmod & Q-Van-leer & Q-Superbee \\
\hline $80 \times 80 \times 80$ & 0.203 & 0.150 & 0.152 & 0.149 & 0.153 \\
\hline
\end{tabular}

All quantities are normalized by $v \Delta \rho / R_{0}^{2}$.

In LES, the grid spacing $\Delta$ is typically much larger than the Kolmogorov length scale so that molecular viscosity plays a negligible role. As the AD resulting from the non-oscillatory convective schemes may affect the SFS motions in LES, we further quantify the dissipation/diffusion property based on SFS turbulence (DMM is used herein, see discussion in Section 2.3). The total SFS 
diffusion, resulting from the unresolvable SGS model and resolvable SFS motion [20], is tabulated in Table VII for all numerical experiments. Both AD and SFS diffusions decrease as the grid interval is decreased. It is important to note that the AD in Table VII is roughly 10 times larger than the amount of SFS diffusion, whereas the SFS turbulent diffusion supposes to dominate the PD of the flow. This suggests that the dissipative property from the monotonic convective schemes dominates the total SFS dissipation in the current simulation, indicating a nearly monotonically integrated LES (MILES). The DMM model adjusts its eddy dissipation/diffusion dynamically to the required amount. MILES uses the features of particular monotonic methods (e.g. ELED schemes in our simulation) to construct implicit SFS models by means of the leading order truncation error [35, 36]. Table VIII compares the averaged AD introduced by different monotonic schemes without SFS modeling (typically MILES) on a high-resolution grid $(80 \times 80 \times 80)$. Our results show that the MILES performs reasonably well in our high-resolution numerical experiments such that the growth rate of instability is accurately captured. The $\mathrm{AD}$ is quantitatively similar to that in Table VI. However, the implicit SFS regularization in MILES results from the non-linearly stable numerical discretization. It is difficult to define a proper filtered-reference solution as the implicitly assumed grid filter is a result of the spatial discretization and highly relies on grid resolution, numerical schemes and flow configuration. This approach is not recommended in the numerical ocean modeling due to its uncertain behavior [37]. This inherent dissipative property applies for all numerical simulations where the monotonic convective schemes are used. We further compare the simulations with a different SGS model in the next section.

\subsection{Comparison of simulations with the Smagorinsky SGS model}

This flow configuration is not only a stringent test for monotonic schemes but also a stringent test for turbulent models. If the modeled SGS stress provides too much energy transfer from large scale to small scale, it will inhibit the growth of upwelling instability [12]. The upwelling experiment is unstable for a range of wavelengths, and there is a wavelength at which the growth rate is maximum. If the initial disturbance contains all wave numbers, the most unstable mode will emerge and dominate the disturbance field. Further, the unsteadiness makes it difficult to differentiate the unresolved SGS flux with the resolvable SFS flux in a global sense. Therefore, we performed additional numerical experiments to examine the effects of the standard Smagorinsky SGS model on the $80 \times 80 \times 80$ grid.

Figure 9 compares the one-dimensional azimuthal energy spectrum at $t=1.31 t_{s}$ for $z=0.9 \mathrm{~h}$ (top) and $z=0.95$ (bottom) at $r=0.85 R$. The energy spectrum from an even higher-resolution simulation (grid size: $96 \times 96 \times 96$ ) is also presented. All energy levels are qualitatively similar except the higher-resolution simulation, which carries slightly larger energy and extends to a broader range. Figure 10 shows the same one-dimensional azimuthal energy spectrum at $r=0.94 R$ (closer to the coastal boundary). It is clear that the energy level is particularly lower for the simulation using the Smagorinsky SGS model. This highly diffusive property can be further illustrated in Figure 11, which shows the salinity contours using Smagorinsky SGS model at $t=1.31 t_{s}$ and $z=0.9 h$. No specific unstable modes emerge in the simulation, so that neither baroclinic instability nor the realistic frontal waves are initialized. This is consistent with the dissipative nature of the Smagorinsky SGS model. Instead, the DMM provides necessary backscatter in energy transfer.

This unrealistic behavior is not surprising. Table IX compares the averaged AD due to the convective scheme with the SFS diffusion based on the Smagorinsky model. The SFS diffusion due to the total SFS turbulence is computed for consistency. The amounts of AD and SFS diffusions 

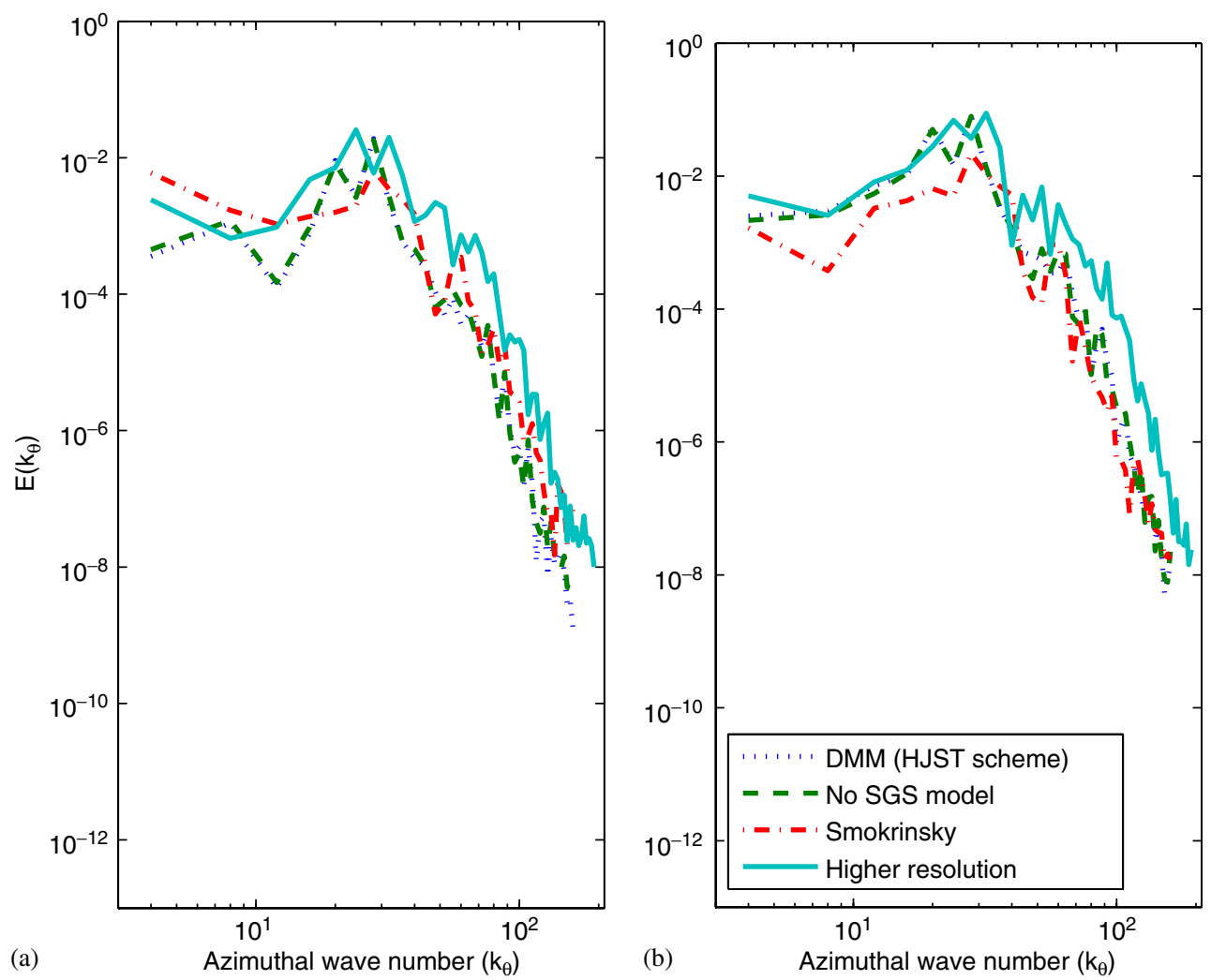

Figure 9. One-dimensional energy spectrum at $t=1.31 t_{s}$ for $z=0.9 h$ (left) and $z=0.95$ (right) at $r=0.85 R$. The grid size for the higher-resolution case is $96 \times 96 \times 96$.

are comparable. More specifically, the calculated total SFS turbulence diffusion is significantly greater than that given by the DMM model. Apparently, this augment directly comes from the constant eddy viscosity coefficient in the Smagorinsky model. The combined effects produce larger diffusion to the upwelling simulation, suppressing the grow of mixed instability. An adequate SFS model is required for realistic simulation and the dynamic approach may compensate the influence of numerical diffusion/dissipation resulting from monotonic convective schemes. Further investigation of the turbulent interaction between the SFS model and numerical discretization error is needed but is beyond the scope of this paper.

\section{CONCLUSIONS}

We developed a new set of higher-order ELED schemes derived from the QUICK formula and alternative lower-order non-oscillatory schemes for unsteady environmental flow simulations. The numerical validations indicate that the best high-order ELED scheme is the Q-JST scheme, which produces very similar results to SHARP. The AD is higher than that introduced by SHARP on coarse grids but is smaller on sufficiently fine grids. The high-order Q-JST scheme performs better 

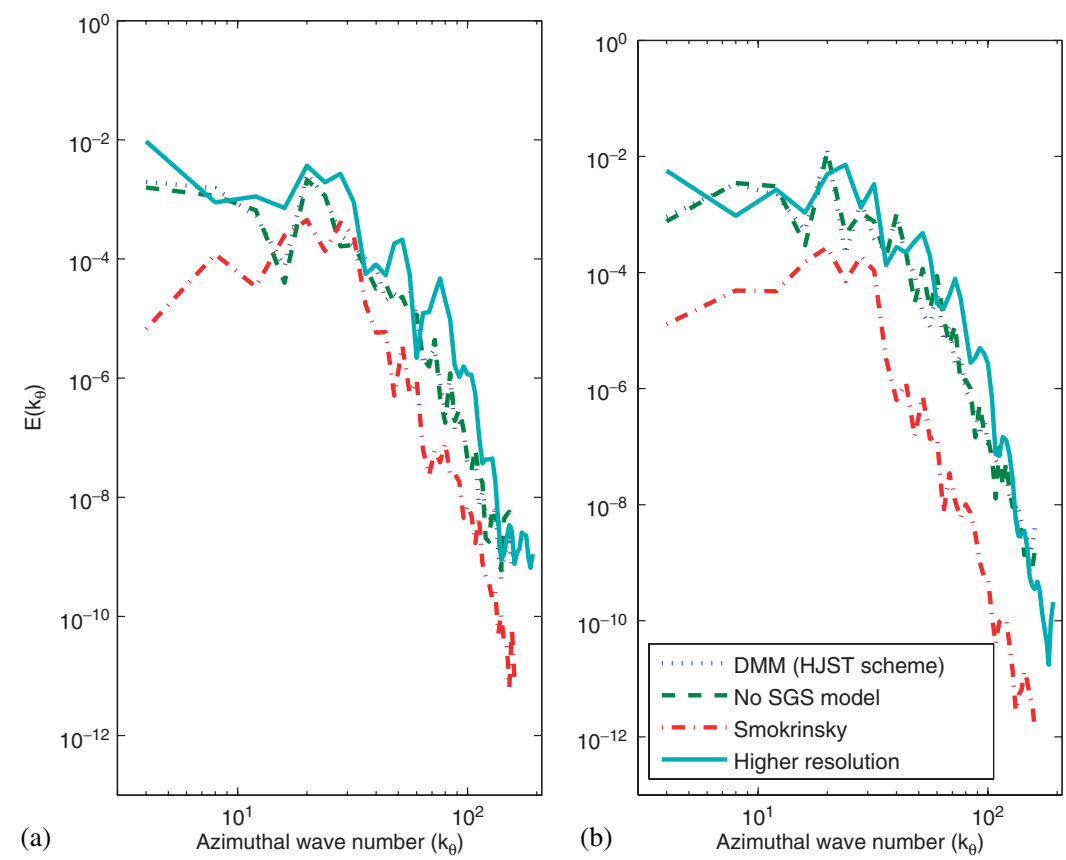

Figure 10. One-dimensional energy spectrum at $t=1.3 t_{s}$ for $z=0.9 h$ (left) and $z=0.95$ (right) at $r=0.94 R$. The grid size for the higher-resolution case is $96 \times 96 \times 96$.

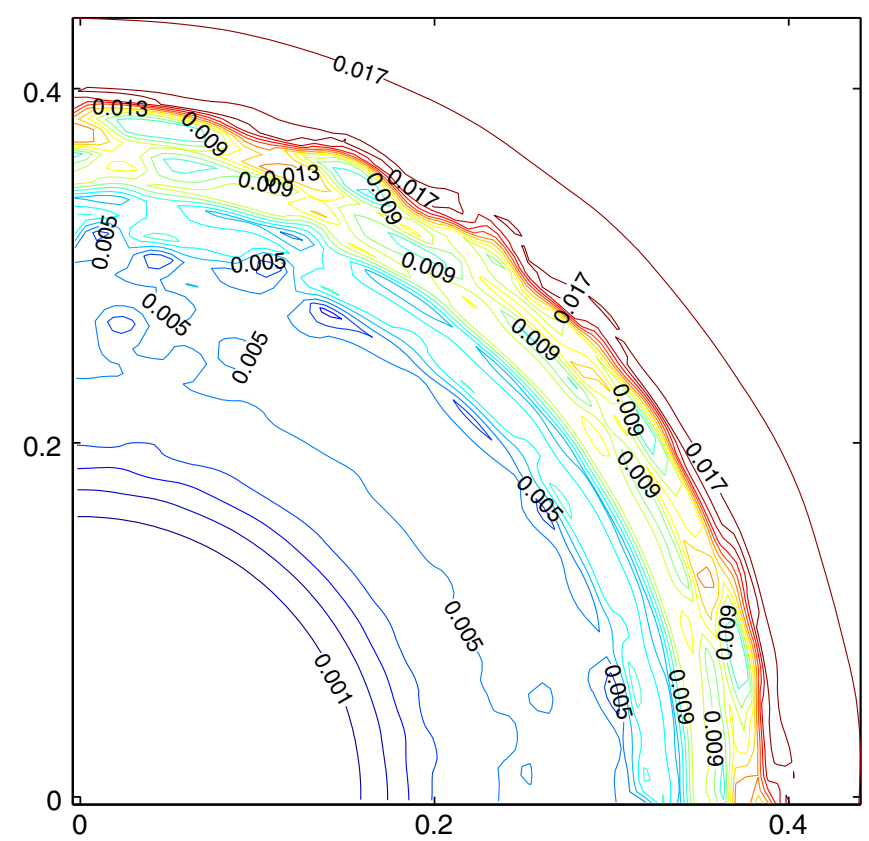

Figure 11. Salinity contours using Smagorinsky SGS model at $t=1.31 t_{s}$ and $z=0.9 h$. 
Table IX. Comparison of the averaged artificial diffusion and SFS diffusion based on the Smagorinsky SGS model.

\begin{tabular}{lcc}
\hline Grid size & Smagorinsky diffusion & Artificial diffusion \\
\hline $64 \times 64 \times 64$ & 0.289 & 0.359 \\
$80 \times 80 \times 80$ & 0.110 & 0.174 \\
\hline
\end{tabular}

All quantities are normalized by $v \Delta \rho / R_{0}^{2}$.

than the other high-order ELED schemes based on SLIP schemes, and the AD is significantly less on fine grids. The ELED treatment does not lose overall accuracy of the based high-order scheme as the switch only adds just enough $\mathrm{AD}$ where it is needed. The amount of $\mathrm{AD}$ can be controlled by the free parameter $\varepsilon$ in Equation (17) and $\beta_{j}$ in Equation (26), which provide more flexibility. In addition, the ELED treatment indeed produces a monotonic scheme, whereas SHARP does not, and it is also less expensive.

In the realistic LES application, the influences of monotonic schemes need careful assessment. The highly diffusive property is still unclear and depends on grid resolution, numerical schemes and flow configuration. A dynamic SFS model can provide proper energy transfer for energy backscatter so that it could potentially compensate the influence of convective schemes. In order to further reduce the additional diffusion in the high-order ELED scheme, we anticipate that, in future work, a higher-order central difference scheme may be preferred to QUICK for the based high-order scheme, as QUICK already introduces a large amount of $\mathrm{AD}$, and additional ELED treatments could add more.

\section{ACKNOWLEDGEMENTS}

The authors would like to thank Dr Parviz Moin and Dr Antony Jameson for their helpful comments and suggestions. The use of the finite-volume code developed at Environmental Fluid Mechanics Laboratory, Stanford is appreciated. Useful comments from the two anonymous reviewers are deeply appreciated. Financial support from National Science Council, Taiwan (grant numbers NSC962628M002010 and NSC962745M002003) is also appreciated.

\section{REFERENCES}

1. Leonard BP. A stable and accurate convective modeling procedure based on quadratic upstream interpolation. Computer Methods in Applied Mechanics and Engineering 1979; 19:58-98.

2. Leonard BP. Beyond first-order upwinding: the ULTRA-SHARP alternative for non-oscillatory steady-state simulation of convection. International Journal for Numerical Methods in Engineering 1990; 30:729-766.

3. Leonard BP. Simple high-accuracy resolution program for convective modelling of discontinuities. International Journal for Numerical Methods in Fluids 1988; 8:1291-1318.

4. Tamamidis P, Assanis DN. Evaluation of various high-order-accuracy schemes with and without flux limiters. International Journal for Numerical Methods in Fluids 1993; 16:931-948.

5. Jameson A. Analysis and design of numerical schemes for gas dynamics 1, artificial diffusion, upwind biasing, limiters and their effect on multigrid convergence. International Journal of Computational Fluid Dynamics 1995; 4:171-218

6. Hirsch C. Numerical Computation of Internal and External Flows. Wiley: New York, 1988.

7. Griffies SM, Pacanowski RC, Hallberg RW. Spurious diapycnal mixing associated with advection in a $z$-coordinate ocean model. Monthly Weather Review 2000; 128:538-564.

8. Ferziger JH, Tseng YH. A coordinate system independent streamwise upwind method for fluid flow computation. International Journal for Numerical Methods in Fluids 2004; 45:1235-1247. 
9. Kunze E, Sandford TB. Abyssal mixing: where it is not. Journal of Physical Oceanography 1996; 26:2286-2296.

10. Zang Y, Street RL. Numerical simulation of coastal upwelling and interfacial instability of a rotating and stratified fluid. Journal of Fluid Mechanics 1995; 305:47-75.

11. Tadepalli S. Numerical simulation and prediction of upwelling flow. Ph.D. Thesis, Stanford University, 1997.

12. Tseng YH, Ferziger JH. Effects of coastal geometry and the formation of cyclonic/anti-cyclonic eddies on turbulent mixing in upwelling simulation. Journal of Turbulence 2001; 2:014.

13. Fringer OB, Armfield SW, Street RL. Reducing numerical diffusion in interfacial gravity wave simulations. International Journal for Numerical Methods in Fluids 2005; 49:301-329.

14. Morinishi Y, Lund T, Vasilyev O, Moin P. Fully conservative higher order finite difference schemes for incompressible flow. Journal of Computational Physics 1998; 143:90-104.

15. Narimousa S, Maxworthy T. Two-layer model of shear-driven coastal upwelling in the presence of bottom topography. Journal of Fluid Mechanics 1985; 159:503-531.

16. Ghosal S. An analysis of numerical errors in large-eddy simulations of turbulence. Journal of Computational Physics 1996; 125:187-206.

17. Zang Y, Street RL, Koseff JR. A dynamic mixed subgrid-scale model and its application to turbulent recirculating flows. Physics of Fluids 1993; A(5):3186-3196.

18. Zang Y. On the development of tools for the simulation of geophysical flows. Ph.D. Thesis, Stanford University, 1993.

19. Germano M. Turbulence: the filtering approach. Journal of Fluid Mechanics 1992; 238:325-336.

20. Chow FK, Street RL, Xue M, Ferziger JH. Explicit filtering and reconstruction turbulence modeling for large-eddy simulation of neutral boundary layer flow. Journal of the Atmospheric Sciences 2005; 62:2058-2077.

21. Smagorinsky J. General circulation experiments with the primitive equations, I. The basic experiment. Monthly Weather Review 1963; 91:99-164.

22. Fringer OB, Armfield SW, Street RL. A nonstaggered curvilinear grid pressure correction method applied to interfacial waves. The Proceedings of 2nd International Conference on Heat Transfer, Fluid Mechanics and Thermodynamics, Victoria Falls, Zambia, 24-26 June 2003.

23. Shyy W, Vu TC. On the adoption of velocity variable and grid system for fluid flow computation in curvilinear coordinates. Journal of Computational Physics 1991; 92:82-105.

24. Zang Y, Street RL, Koseff JR. A non-staggered grid, fractional step method for time-dependent incompressible Navier-Stokes equations in curvilinear coordinates. Journal of Computational Physics 1994; 114:18-33.

25. Tseng YH, Ferziger JH. Large-eddy simulation of turbulent wavy boundary flow-illustration of vortex dynamics. Journal of Turbulence 2004; 5:34.

26. Kim J, Moin P. Application of a fractional-step method to incompressible Navier-Stokes equations. Journal of Computational Physics 1985; 59:308-323.

27. Choi HG, Choi H, Yoo JY. A fractional four-step finite element formulation of the unsteady incompressible Navier-Stokes equations using SUPG and linear equal-order element methods. Computer Methods in Applied Mechanics and Engineering 1997; 143:333-348.

28. Fringer OB, Street RL. The dynamics of breaking progressive interfacial waves. Journal of Fluid Mechanics $2003 ;$ 494:319-353.

29. Venayagamoorthy SK, Fringer OB. Numerical simulations of the interaction of internal waves with a shelf break. Physics of Fluids 2006; 18. DOI:10.1063/1.2221863.

30. Jameson A, Schmidt W, Turkel E. Numerical solutions of the Euler equations by finite volume methods with Runge-Kutta time stepping schemes. AIAA Paper 81-1259, 1981.

31. Fringer OB. Numerical simulation of breaking interfacial waves. Ph.D. Thesis, Stanford University, 2001.

32. Thorpe SA. On standing internal gravity waves of finite amplitude. Journal of Fluid Mechanics 1968; 32:693-704.

33. Linden PF, Van Heijst JF. Two-layer spin-up and frontogenesis. Journal of Fluid Mechanics 1984; 143:69-94.

34. Tseng YH, Ferziger JH. Mixing and available potential energy in stratified flows. Physics of Fluids 2001; 13:1281-1293.

35. Boris JP, Grinstein FF, Oran ES, Kolbe RL. New insights into large eddy simulation. Fluid Dynamics Research 1992; 10:199-228.

36. Grinstein FF, Fureby C. Recent progress on miles for high Reynolds number flows. Journal of Fluids EngineeringTransactions of the ASME 2002; 124:848-861.

37. Tseng YH, Dietrich DE. Entrainment and transport in idealized three-dimensional gravity current simulation. Journal of Atmospheric and Oceanic Technology 2006; 23:1249-1269. 